\title{
Game Design Considerations When Using Non-touch Based Natural User Interface
}

\author{
Mohd Fairuz Shiratuddin and Kok Wai Wong \\ School of Information Technology \\ Murdoch University \\ South Street, Murdoch, Western Australia 6150 \\ \{f.shiratuddin, k.wong\} @murdoch.edu.au
}

Extended Abstract. In recent years, the advancement in gaming interface has paved ways for faster and more interactive gameplay. There is now an increasing trend of using Natural User Interface (NUI) for computer games. This has also brought forward new challenges in game design and using non-touch based NUI in games is not simply replacing the existing interaction techniques. A lot of new game design considerations must be carefully thought out, planned and implemented, or otherwise the gameplay will suffer and, could cause frustration and dissatisfaction to the players. This paper discusses non-touch based Natural User Interface (NUI) and the factors that need to be considered by designers of games in designing games that uses this new user interface. Many considerations must be addressed to create the effective user experience when using NUI. This paper provides a framework of game design considerations that can be classified into three main categories: (1) user based analysis, (2) function based analysis, and (3) ambient based analysis. In this paper the following considerations have been identified and they fall under these three main categories:

1) User based analysis

- Target Audience

- Genre

2) Function based analysis

- $\quad$ Players' Perceived Playability

- Gaming Space Requirement

- Suitable Gestures

- Gestural Control

- Multi-Player/Single Player

3) Ambient based analysis

- $\quad$ Speech Control \& Audio Elements

- Iconography

- Types of Display Screen

Since the use of NUI is a new phenomenon in games, the design considerations presented in this paper are by no means exhaustive. However, the discussions provided in this paper can reveal potential areas for future research in the field of nontouch based NUI for games. 